OnLine Journal of Biological Sciences 12 (1): 11-14, 2012

ISSN 1608-4217

(C) 2012 V.P. Singh et al., This open access article is distributed under a Creative Commons Attribution

(CC-BY) 3.0 license

\title{
Focal Adhesion Kinase: An Old Protein with New Roles
}

\author{
${ }^{1}$ Atul Kumar Pandey, ${ }^{2}$ Sonal Somvanshi and ${ }^{2}$ Vivek Pratap Singh \\ ${ }^{1}$ Department of Biology, West Virginia State University, Institute, West Virginia, \\ ${ }^{2}$ Department of Pathology and Laboratory Medicine, \\ ${ }^{3}$ Department of Pharmacology and Cell Biophysics, \\ University of Cincinnati, College of Medicine, Cincinnati, Ohio
}

\begin{abstract}
Focal adhesion kinase, FAK is a tyrosine kinase which is prominently localized to focal adhesions and therefore its name, is an indispensable protein in integrin signaling. Since the discovery of FAK, this tyrosine kinase had been shown to regulate a variety of cell behavior like cell migration, proliferation, apoptosis and metastasis which makes this molecule at a centre stage of cell and developmental biology research. We want to take this opportunity to briefly review the current state of knowledge about FAK and why FAK was so important in cell migration, apoptosis, cardiovascular and metastatic research. Since the FAK signalling was very crucial for normal cellular development and cell physiology and since its deregulation prompts the oneset of a variety of diseases like cancer and cardiovascular diseases, an updated knowledge about its signalling mechanism and how FAK interacts with other signalling molecules can not only offer newer understanding in this field but also attract new methods and pathways to explore and investigate its biology, which can further open new avenues in anticancer research targetting FAK.
\end{abstract}

Key words: FAK, cell migration, angiogenesis and cancer

\section{INTRODUCTION}

Focal Adhesion Kinase (FAK) is a cytoplasmic protein tyrosine kinase that plays an important role during embryonic development and in different diseases including cancer and cardiovascular disorders (Golubovskaya et al., 2009). Basically, FAK is a 125$\mathrm{kDa}$ protein tyrosine kinase which is made up of an $\mathrm{N}$ terminal FERM domain, a central kinase domain, two proline-rich motifs and a C-terminal focal adhesion targeting, FAT domain, as shown in Fig. 1 (Girault et al., 1999; Sun et al., 2002).

The most important event in FAK signaling is the phosphorylation of Tyr397 (Schaller et al., 1994; Lai et al., 2010), which eventually promotes the recruitment and activation of $\mathrm{Src}$ to cell-extracellular matrix adhesion, sites (Yeo et al., 2006). This Tyr397 phosphorylation can then trigger the phosphorylation and activation of a variety of other kinases including its own tyrosine kinase residue FAK Tyr576/Tyr577 which promotes maximal FAK catalytic activity (CaronLormier and Berry, 2005). The phosphorylation of FAK Tyr925 located in the FAT domain is probably a late event but recognized as one of the several mechanisms through which adhesion can promote the activation of Ras/mitogen-activated protein kinase pathway (Mitra et al., 2005). FAK also has a paxillin binding site, which is considered as a mediator of FAK binding to integrins in the process of formation of adhesion complexes. Largely, the FAK signaling is explained in terms of its interaction with integrins, however, several growth factors like EGFRs, chemokines and G-protein coupled receptors also interacts and activate the FAK signaling, therefore it seems like FAK activation is a secondary event and the result from other pathways affecting integrin function. The complete mechanism of FAK signaling and its downstream signaling has been reviewed in several recent articles, therefore, we want to briefly emphasize the various role of FAK and will focus on how FAK could be an attractive target in anticancer research and beyond.

FAK and cell migration: Cell migration is a coordinated and complex process and evidence indicates the involvement of FAK in cell migration (Erickson, 1990). FAK signaling enhances cell motility, whereas inhibition of FAK signaling impairs cell migration (Mitra et al., 2005).

Corresponding Author: Vivek Pratap Singh, Department of Pharmacology and Cell Biophysics,

University of Cincinnati College of Medicine, 231 Albert Sabin Way Cincinnati,

Ohio Tel: 45267-0575 513-558-2327 Fax: 513-558-2269 
OnLine J. Biol. Sci., 12 (1): 11-14, 2012

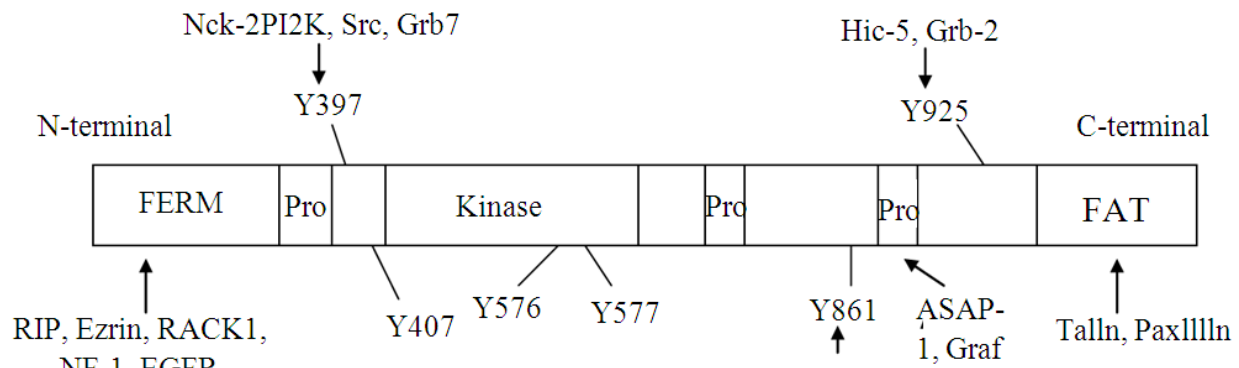

Fig. 1:Structure of Focal Adhesion Kinase and interaction proteins. FAK has N-terminal, central kinase and Cterminal domains. There are several known phosphorylation sites and interacting partners, indicated in the figure

FAK regulates the motility in randomly migrating cells and in response to a broad range of stimuli, including chemotactic, haptotactic and durotactic signals. Embryonic cells from FAK-deficient mice exhibited a decreased migration in culture, which was suggested to be responsible for a defect in mesodermal migration resulting in an embryonic lethal phenotype of the FAKdeficient ice, providing direct evidence for a role of FAK in promoting migration (Ilic et al., 1995). In vivo, microinjection of the FAK C-terminal recombinant protein reduced FAK activation and reduced migration of fibroblasts (Gilmore and Romer, 1996). There are reports showing FAK promote cell migration trough its interactions with PI3K and an adaptor molecule Grb7 (Han and Guan , 1999; Han et al., 2000). Similarly, expression of FAT, FRNK, or mutant FAK (397F) constructs, which typically act as dominant interfering forms of FAK, in various cell types usually results in partial inhibition of FAK activation and partial inhibition of cell migration (Hauck et al., 2001; Ding et al., 2005). Conversely, the overexpression of wild-type FAK in various different cell types enhances cell migration (Wang et al., 2000).

FAK and cell survival: Cell growth and survival is a very tightly regulated process and the cell fate is always a pre-determined event. The mechanism of apoptosis insures that the senescent and dead cells are cleared and this process is highly regulated. However this normal mechanism of apoptosis is also the target of tumor promoting factors, proto-oncogenes and several kinases that are up regulated in tumor cells. In fact this normal mechanism of apoptosis is actually hijacked to promote cell growth without inhibition. FAK knockout in mouse endothelial cells have shown to cause vascular defects and an increase in cell apoptosis and aberrant cell migration (Braren et al., 2006).

Several pathways downstream of FAK could be involved in cell survival. A phosphorylated FAK at
Tyr397 can also bind to the p85 subunit of phosphatidylinositol 3-kinase, PI-3K and the production of phospholipids by phosphatidylinositol 3-kinase can lead to the activation of Akt kinase that inhibits apoptosis by regulating various components of the cell death machinery. The role of FAK have been investigated to promote cell proliferation by suppressing the process of apoptosis and also by some other mechanisms like activation of c-Jun NH2terminal kinase downstream of CAS (Almeida et al., 2000). FAK therefore acts like a double edge sword where it's knocking down cause's severe defects in cell motility and embryonic lethality and on the other side this molecule is involved in suppressing apoptosis and therefore acts like a tumor promoter kinase.

FAK in cardiovascular development: Recent studies using animal models have shown an important role for FAK in the cardiovascular physiology. In particular, FAK is essential for angiogenesis in the embryo, functions in heart development and modulates the response of cardiomyocytes to pressure overload in adult mice. FAK inactivated mice were dead within hours of birth, mainly due to defective heart function and incomplete formation of the septum (Hakim et al., 2007). FAK gene knockout in mouse model have shown early embryonic lethality and severe cardiovascular defects (Ilic et al., 1995). In FAK conditional knockout mice there are defects in cardiac developmental and eccentric right ventricular hypertrophy in cardiomyocyte (Peng et al., 2008). FAK also play a modifying role in cardiac hypertrophy. In vivo, loss of FAK expression resulted in increased dilation of the ventricle lumen upon AngII induced hypertrophy and aortic banding. This study suggests that Inactivation of focal adhesion kinase in cardiomyocytes promotes eccentric cardiac hypertrophy and fibrosis (Peng et al., 2006). 
FAK in angiogenesis: Angiogenesis, the formation of new blood vessels from pre-existing ones, is essential for tumor development. Several angiogenic growth factor receptors regulate FAK expression. A number of studies in recent days have shown direct evidence about the role of FAK in angiogenesis utilizing transgenic and knockout mouse model system. Global mutant FAKknockin mouse model reporting, specific roles for the kinase activity of FAK in embryonic vessel development. Lim et al. (2010) generated a knockin mouse model in which a FAK utation abolished the catalytic activity of the kinase domain. They reported that the inhibition of FAK kinase activity led to a lethal embryonic phenotype at E9.5, associated with hemorrhage and damage of blood vessels (Lim et al., 2010). FAK has been shown as an important modulator of angiogenesis, as transgenic mouse models have indicated that endothelial FAK expression and activity are essential for the formation of new blood vessel networks during embryonic development (Braren et al., 2006). Recently, using a tissue-restricted knockout mouse model, it was demonstrated that endothelial FAK was essential for tumor growth and tumorassociated angiogenesis, as mice lacking endothelialspecific FAK expression exhibited reduced tumor angiogenesis and hence reduced tumor growth in vivo (Tavora et al., 2010). In addition to its putative role in angiogenesis, altered FAK activity and expression have been directly linked to tumor genesis and metastasis since interference with FAK signaling led to decreased metastasis in a variety of tumor models, including breast and lung cancer (Golubovskaya et al., 2009; Zhao and Guan, 2009).

Targeting FAK in cancer: future direction in FAK research: FAK is an important regulator of cell migration and angiogenesis that is why FAK may be a best target in different diseases those that are very dependent on these biological process. A good example will be cancer metastasis where both of these processes involved. Pharmacological inhibition of FAK is one of the several exciting area in anticancer research as the FAK activity have been found up regulated in a variety of cancers such as ovarian cancer, neck, colon, prostate, thyroid and many other cancer types (McLean et al., 2005; Brunton and Frame, 2008). Therefore there is a big surge in research targeting FAK as a possible means to suppress the tumor cells. Several small molecule inhibitors are under way at various stages and few are undergoing clinical trials for cancer treatment (McLean et al., 2012). Several lines of evidence in cultured cells and conditional knock-out mouse models have suggested a critical role of FAK in angiogenesis during embryonic development and tumorigenesis (Lechertier and Hodivala-Dilke, 2012). Pfizer has developed a FAK specific inhibitor, PF-573,228 and another dual inhibitor PF-562,271, targeting FAK and a related molecule Pyk2, to check phosphorylation of FAK Tyr397. However the strategy is not as simple as it sounds because most of the inhibitors so far have targeted only the catalytic activity of FAK thereby targeting Tyr397 and suppressing the FAK-Src complex formation. But the kinase-independent functions of FAK observed in endothelial cells and mouse embryonic fibroblasts may also be true in cancer cells, therefore the small molecule inhibitors specifically designed to target both kinase dependent and kinaseindependent functions of FAK along with targeting FAk's interaction with other molecules and pathways may prove much more promising. Another line of research could be two separate investigations, each targeting for kinase dependent and independent functions of FAK and then trying both inhibitors in combination. Much more reproducible results in different cancer cell types and mouse models would be needed before we reach to any conclusion and therefore we observe at this stage that both kinase dependent and independent inhibition of FAK will remain an active area of investigation in search of better anticancer drugs for the future.

\section{REFERENCES}

Almeida, E.A., D. Ilic, Q. Han, C.R. Hauck and F. Jin et al., 2000. Matrix survival signaling: From fibronectin via focal adhesion kinase to c-Jun NH(2)-terminal kinase. J Cell Biol., 149: 741-54. PMID: 10791986

Braren, R., H. Hu, Y.H. Kim, H.E. Beggs and L.F. Reichardt et al., 2006. Endothelial FAK is essential for vascular network stability, cell survival and lamellipodial formation. J. Cell. Biol., 72: 151-62. PMID: 16391003

Brunton, V.G. and M.C. Frame, 2008. Src and focal adhesion kinase as therapeutic targets in cancer. Curr. Opin. Pharmacol., 8: 427-432. PMID: 18625340

Caron-Lormier, G. and H. Berry, 2005. Amplification and oscillations in the FAK/Src kinase system during integrin signaling. J. Theor. Biol., 232: 23548. PMID: 15530493

Ding, Q., J.R. Grammer, M.A. Nelson, J.L. Guan and J.E. Stewart Jr. et al., 2005. p27(Kip1) and cyclin D1 are necessary for Focal Adhesion Kinase (FAK) regulation of cell cycle progression in glioblastoma cells propagated in vitro and in vivo in the scid mouse brain. J. Biol. Chem., 280: 68026815. PMID: 15557280 
Erickson, C.A., 1990. Cell migration in the embryo and adult organism. Curr. Opin. Cell Biol., 2: 67-74. PMID: 2183845

Gilmore, A.P. and L.H. Romer., 1996. Inhibition of Focal Adhesion Kinase (FAK) signaling in focal adhesions decreases cell motility and proliferation. Mol. Biol. Cell., 7: 1209-1224. PMID: 8856665

Girault, J.A., G. Labesse, J.P. Mornon and I. Callebaut, 1999. The N-termini of FAK and JAKs contain divergent band 4.1 domains. Trends Biochem. Sci., 24: 54-57. PMID: 10098398

Golubovskaya, V.M., F.A. Kweh and W.G. Cance, 2009. Focal adhesion kinase and cancer. Histol. Histopathol., 24: 503-510. PMID: 19224453

Hakim, Z.S., L.A. DiMichele, J.T. Doherty and J.W. Homeister et al., 2007. Conditional deletion of focal adhesion kinase leads to defects in ventricular septation and outflow tract alignment. Mol. Cell Biol., 27: 5352-5364. PMID: 17526730

Han, D.C. and J.L. Guan, 1999. Association of focal adhesion kinase with Grb7 and its role in cell migration. J. Biol. Chem., 274: 24425-24430. PMID: 10446223

Han, D.C., T.L. Shen and J.L. Guan, 2000. Role of Grb7 targeting to focal contacts and its phosphorylation by focal adhesion kinase in regulation of cell migration. J. Biol. Chem., 275: 28911-28917. PMID: 10893408

Hauck, C.R., D.J. Sieg, D.A. Hsia, J.C. Loftus and W.A. Gaarde et al., 2001. Inhibition of focal adhesion kinase expression or activity disrupts epidermal growth factor-stimulated signaling promoting the migration of invasive human carcinoma cells. Cancer Res., 61: 7079-7090. PMID: 11585739

Ilic, D., Y. Furuta, S. Kanazawa, N. Takeda and K. Sobue et al., 1995. Reduced cell motility and enhanced focal adhesion contact formation in cells from FAK-deficient mice. Nature., 377: 539-544. PMID: 7566154

Lai, I.R., P.Y. Chu, H.S. Lin, J.Y. Liou and Y.J. Jan et al., 2010. Phosphorylation of focal adhesion kinase at Tyr397 in gastric carcinomas and its clinical significance. Am. J. Pathol., 177: 1629-37. PMID: 20724588

Lechertier, T. and K. Hodivala-Dilke, 2012. Focal adhesion kinase and tumour angiogenesis. J. Pathol., 226: 404-412. DOI: 10.1002/path.3018

Lim, S.T., X.L. Chen, A. Tomar, N.L. Miller and J. Yoo et al., 2010. Knock-in mutation reveals an essential role for focal adhesion kinase activity in blood vessel morphogenesis and cell motilitypolarity but not cell proliferation. J. Biol. Chem., 285: 21526-21536. PMID: 20442405
McLean, G.W., N.O. Carragher, E. Avizienyte J. Evans and V.G. Brunton et al., 2005. The role of focaladhesion kinase in cancer, a new therapeutic opportunity. Nat Rev Cancer., 5: 505-515. PMID: 16069815

McLean, G.W., N.O. Carragher, E. Avizienyte, J. Evans and V.G. Brunton et al., 2012. The role of focal-adhesion kinase in cancer, a new therapeutic opportunity. Nat. Rev. Cancer, 5: 505-515. PMID: 16069815

Mitra, S.K., D.A. Hanson and D.D. Schlaepfer, 2005. Focal adhesion kinase: In command and control of cell motility. Nat. Rev. Mol. Cell. Biol., 6: 56-68. PMID: 15688067

Peng, X., M.S. Kraus, H. Wei, T.L. Shen and R. Pariaut et al., 2006. Inactivation of focal adhesion kinase in cardiomyocytes promotes eccentric cardiac hypertrophy and fibrosis in mice. J. Clin. Invest., 116: 217-227. PMID: 16374517

Peng, X., X. Wu, J.E. Druso, H. Wei, A.Y. Park et al., 2008. Cardiac developmental defects and eccentric right ventricular hypertrophy in cardiomyocyte Focal Adhesion Kinase (FAK) conditional knockout mice. Proc. Natl. Acad. Sci. U S A., 6: 105: 6638-6643. PMID: 18448675

Schaller, M.D., J.D. Hildebrand, J.D. Shannon, J.W. Fox and R.R. Vines et al., 1994. Autophosphorylation of the focal adhesion kinase, pp125FAK, directs SH2-dependent binding of pp60src. Mol. Cell. Biol., 14: 1680-1688. PMID: 7509446

Sun, C.X., V.A. Robb and D.H. Gutmann, 2002. Protein 4.1 tumor suppressors: Getting a FERM grip on growth regulation. J. Cell Sci., 115: 39914000. PMID: 12356905

Tavora, B., S. Batista, L.E. Reynolds, S. Jadeja and S. Robinson et al., 2010. Endothelial FAK is required for tumour angiogenesis. EMBO Molecular Med., 2: 516-528. PMID: 21154724

Wang, D., R. Grammer, C.S. Cobbs, J.E. Stewart Jr and Z. Liu et al., 2000. p125 focal adhesion kinase promotes malignant astrocytoma cell proliferation in vivo. J. Cell Sci., 113: 4221-4230. PMID: 11069767

Yeo, M.G., M.A. Partridge, E.J. Ezratty, Q. Shen and G.G. Gundersen et al., 2006. Src SH2 arginine 175 is required for cell motility: Specific focal adhesion kinase targeting and focal adhesion assembly function. Mol. Cell Biol., 26: 4399-409. PMID: 16738308

Zhao, J. and J.L. Guan, 2009. Signal transduction by focal adhesion kinase in cancer. Cancer Metastasis Rev., 28: 35-49. PMID: 19169797 